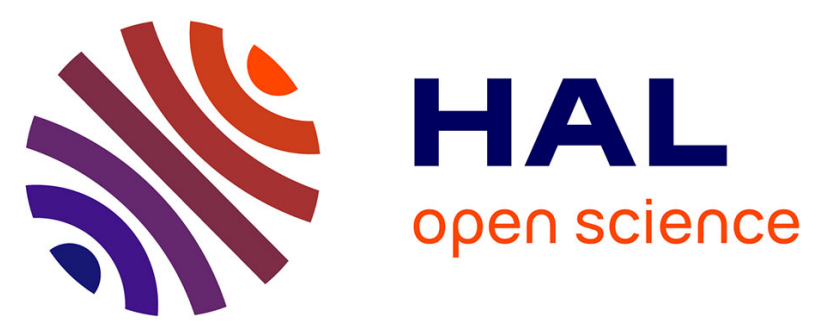

\title{
Preliminary results for introducing dependent random variables in stochastic feasibility analysis on CAN \\ Liliana Cucu
}

\section{To cite this version:}

Liliana Cucu. Preliminary results for introducing dependent random variables in stochastic feasibility analysis on CAN. 7th IEEE International Workshop on Factory Communication Systems - WFCS'08, Work-in-Progress session, May 2008, Dresden, Germany. pp.271-274, 10.1109/WFCS.2008.4638759 . inria-00334693

\section{HAL Id: inria-00334693 \\ https://hal.inria.fr/inria-00334693}

Submitted on 27 Oct 2008

HAL is a multi-disciplinary open access archive for the deposit and dissemination of scientific research documents, whether they are published or not. The documents may come from teaching and research institutions in France or abroad, or from public or private research centers.
L'archive ouverte pluridisciplinaire HAL, est destinée au dépôt et à la diffusion de documents scientifiques de niveau recherche, publiés ou non, émanant des établissements d'enseignement et de recherche français ou étrangers, des laboratoires publics ou privés. 


\title{
Preliminary results for introducing dependent random variables in stochastic feasibility analysis on CAN
}

\author{
Liliana Cucu, TRIO, LORIA-INPL \\ Villers-les-Nancy, France \\ liliana.cucu@loria.fr
}

\begin{abstract}
We consider periodic and sporadic messages that are scheduled on a Controller Area Network (CAN) bus. We propose a stochastic analysis framework that provides probability distributions of message response times where the arrival of messages are given by independent random variables. This analysis can be extended easily to the case of dependent random variables. We also define the necessary steps to validate such analysis.
\end{abstract}

\section{Introduction}

Context of the study Requests in real-time environment are often of a recurring nature. Such systems are typically modeled as finite collections of simple, highly repetitive activities (e.g., tasks, messages). When the different instances of those activities are generated in a very predictable manner, we deal with periodic activities. The real-time performances of periodic activities on uniprocessor, distributed or network systems have been extensively studied. For these activities, the corresponding response times can be evaluated when all their parameters are known, but this is not the case of all real-time applications. Some parameters can be unknown until the time instant when the activity is released or the environment can change forcing the application to adapt. For instance, the problem is quite different for sporadic activities since, in many practical cases, it is not possible to have a precise knowledge of the activation pattern before the implementation of the system. For instance this is the case for sporadic messages, usually subject to soft real-time constraints, exchanged among Electronic Control Units (ECUs) in the body network of a vehicle. Such messages are usually assigned a low priority and they do not delay the hard real-time periodic traffic. However it is difficult to estimate their own response times.

Problem definition In this paper we discuss the problem of evaluating the real-time performances of periodic and sporadic messages that are scheduled on a CAN bus. We deal in this context with nonpreemptive fixed-priority scheduling under the hypothesis that each message has its own priority. We are interested in obtaining probability distribution functions for the response times of messages, be they periodic or sporadic. We are clearly here in the realm of soft real-time constraints, but their satisfaction is important since large response times may jeopardize the execution of a function and may even raise safety concerns in some cases. In addition, low responsiveness is negatively perceived by the user.

Existing work Our approach belongs to the class of stochastic analyses for real-time systems. Probabilistic approaches are promising because they answer questions that cannot be addressed in a deterministic manner (e.g., distribution of response times) and consider models that are more realistic for instance regarding the task activation patterns or the expression of soft real-time constraints.

These approaches can be classified in two main classes. One class consists in extracting quantitative information for one or more parameters (e.g. distribution of execution time) from samples of observations collected by monitoring the system [1,2]. Such approaches actually belong more to the realm of statistical methods. The other class of stochastic approaches concerns the temporal analysis of systems that have at least one parameter being a random variable. Among the studies in this area, one can for instance mention [3, 4].

To our best knowledge, there is no study on the response time of CAN-based systems for the case where the arrival of sporadic messages are given by independent random variables, which is the focus of this paper. 
Our contribution Based on the exact response time analysis given in [5], we give a stochastic equation for calculating the distribution functions of messages response times on CAN. This equation can be solved using an algorithm provided in [4]. Moreover, it can be easily extended to dependent random variables by replacing the convolutions with the corresponding operations defined in [6]. These operations are based on the introduction of copulas, which to our best knowledge has never been used in this context. Copulas have been introduced to real-time systems in a seminal paper of Bernat et al. [6] and this theory allows to take into account possible dependencies between the random variables giving the inter-arrival times.

Organisation of the paper The paper is organized as follows. Section 2 contains our model and the associated notations. In Section 3 we introduce existing results that we use in this paper. In Section 4 we propose a stochastic feasibility analysis and in Section 5 necessary steps to validate a stochastic analysis. We present some ideas on future work in Section 6.

\section{Model and notations}

We analyse here the response time of messages under non-preemptive fixed-priority scheduling on a CAN bus. We consider $\tau=\left\{\tau_{1}, \tau_{2}, \cdots, \tau_{n}\right\}$ a set of messages, ordered in the decreasing order of their priority.

Each message is characterized by a transmission time $C_{i}$ and a relative deadline $D_{i}$. Each new release $j$ of a message $\tau_{i}$ is defined by different random variables. We denote by $\mathcal{T}_{i, j}{ }^{1}$ the random variable giving the inter-arrival time between the $(j)^{t h}$ and the $(j+1)^{t h}$ instance of a message $\tau_{i}$. The first release happens at a time instant given by $\mathcal{T}_{i, 0}$. It is assumed that all random variables are independent of other jobs of this message.

To facilitate the presentation, we consider $\mathcal{T}_{i, j}$ to be discrete, thus its probability mass function (pmf) is:

$$
\mathcal{T}_{i, j}=\left(\begin{array}{c}
t_{k} \\
P\left(T=t_{k}\right)
\end{array}\right)_{k \in\left\{1, \cdots, k_{i, j}\right\}}
$$

where $t_{k} \in\left[t_{i}^{\min }, t_{i}^{\max }\right]$ and $k_{i, j} \in \mathbb{N}^{*}$ is the number of values that random variable $\mathcal{T}_{i, j}$ has. We consider that $t_{i}^{\text {min }}, t_{i}^{\max }$ are given, thus known. If $t_{i}^{\text {min }}=$

\footnotetext{
${ }^{1}$ In this paper we utilise calligraphic letters to denote random variables
}

$t_{i}^{\max }=T_{i}$ then the message $\tau_{i}$ is a periodic message and we have $\mathcal{T}_{i, j}=\left(\begin{array}{c}T_{i} \\ 1\end{array}\right)$. If $t_{i}^{\text {min }} \neq t_{i}^{\text {max }}$, then the message $\tau_{i}$ is a random message.

We consider in this paper that $\sum_{i=1}^{n} \frac{C_{i}}{t_{i}^{\min }} \leq 1$.

The set $h p(i)$ contains the indices of messages with higher priority than $\tau_{i}$ and $l p(i)$ the indices of messages with lower priority than message $\tau_{i}$. The set $h p(i)$ contains $\mathbf{R}(i)$ which is the set of indices of random higher priority messages and $\mathbf{P}(i)$ the set of indices of periodic higher priority messages.

All considered values are multiples of $\tau_{b i t}$ which is the time duration of a single bit on the CAN bus.

\section{Existing results that we use}

\subsection{Deterministic exact analysis for CAN}

In this section, since we present existing results for periodic messages, we consider that each message $\tau_{i}$ is characterized by its minimal inter-arrival time $T_{i}$.

We present here existing results [5] giving the worst-case response time of a message $\tau_{i}$ when all messages are periodic. First we need to calculate the length of the busy period for a message of priority $i$. We can obtain the length by solving iteratively the following equation

$$
t_{i}=B_{i}+\sum_{k \in h p(i) \cup\{i\}}\left\lceil\frac{t_{i}}{T_{k}}\right\rceil C_{k}
$$

where $B_{i}=\max _{k \in l p(i)} C_{k}$.

Then the worst-case response time of $\tau_{i}$ is obtained as

$$
R_{i}=\max _{q=0 \ldots Q_{i}-1}\left\{W_{i}(q)-q T_{i}+C_{i}\right\}
$$

where $Q_{i}=\left\lceil\frac{t_{i}}{T_{i}}\right\rceil$ and $W_{i}(q)$ is the solution of the following equation:

$$
W_{i}(q)=B_{i}+q C_{i}+\sum_{k \in h p(i)}\left\lceil\frac{W_{i}(q)+\tau_{b i t}}{T_{k}}\right\rceil C_{k}
$$

Equation (4) can be solved iteratively by considering at the first step of the iteration $W_{i}(q)^{0}=0$.

\subsection{Stochastic analysis for preemptive uniprocessor fixed-priority scheduling}

We present here existing stochastic equation giving the response times distribution of activities un- 
der preemptive uniprocessor fixed-priority scheduling [4].

When the set of activities is strictly composed of periodic ones, then the following well-known formulation allows computing the worst-case response time of activity $\tau_{i}$ (we consider the activities ordered in the decreasing order of their priorities):

$$
R_{i}=C_{i}+\sum_{k \in h p(i)}\left\lceil\frac{R_{i}}{T_{k}}\right\rceil C_{k}
$$

Equation (5) can be solved iteratively, by forming a recurrence relationship. This equation was extended to the case of activities with inter-arrival times given by independent random variables [4]:

$\mathcal{R}_{i}=\mathcal{C}_{i} \otimes\left(\otimes_{k \in \mathbf{P}(i)}\left\lceil\frac{\mathcal{R}_{i}}{T_{k}}\right\rceil C_{k}\right) \otimes\left(\otimes_{k \in \mathbf{R}(i)} \mathcal{N}_{\tau_{k}}\left(\mathcal{R}_{i}\right) C_{k}\right)$

where $\mathcal{C}_{k}=\left(\begin{array}{c}C_{k} \\ 1\end{array}\right), \forall k \in \mathbb{N}, \mathcal{C}_{i}=\left(\begin{array}{c}C_{i} \\ 1\end{array}\right), \forall i \in$ $\mathbb{N}$ and $\mathcal{N}_{\tau_{k}}\left(\mathcal{R}_{i}\right)$ is the random variable giving the number of arrivals of $\tau_{k}$ with their probabilities within $\mathcal{R}_{i}$. By $\otimes$ we understand the convolution of two independent random variables.

\section{Stochastic feasibility analysis}

Without loss of generality and in order to facilitate the presentation, we consider that $\mathcal{T}_{i, j}=\mathcal{T}_{i, k}, \forall j, k$.

For each message $\tau_{k} \in \mathbf{R}(i)$ we take into account the smallest values $t_{k}^{\min }$ in Equation (2) and we obtain the following formulation:

$$
t_{i}=B_{i}+\sum_{k \in h p(i) \cup\{i\}}\left\lceil\frac{t_{i}}{t_{k}^{\min }}\right\rceil C_{k}
$$

We extend now the response time formulation to the case with random variables by replacing max with the mean in Equation ( 3$)^{2}$. We obtain that the average response time of $\tau_{i}$ is obtained as the distribution function:

$$
\mathcal{R}_{i}=\frac{1}{q} \sum_{q=0 \ldots Q_{i}-1}\left\{\mathcal{W}_{i}(q) \otimes\left(\begin{array}{c}
-q t_{i}^{\min } \\
1
\end{array}\right) \otimes \mathcal{C}_{i}\right\}
$$

where $Q_{i}=\left\lceil\frac{t_{i}}{t_{i}^{m i n}}\right\rceil$ and $\mathcal{W}_{i}(q)$ is the solution of the following equation:

\footnotetext{
${ }^{2}$ One can choose other way of merging the $q$ response times that corresponds better to his/her system.
}

$$
\begin{array}{r}
\mathcal{W}_{i}(q)=\left(\otimes_{k \in \mathbf{P}(i)}\left\lceil\frac{\mathcal{W}_{i}(q)+\tau_{b i t}}{\mathcal{T}_{k}}\right\rceil \mathcal{C}_{k}\right) \otimes \\
\left(\otimes_{k \in \mathbf{R}(i)} \mathcal{N}_{K}\left(\mathcal{W}_{i}(q)\right) \mathcal{C}_{K}\right) \otimes\left(\begin{array}{c}
B_{i}+q C_{i} \\
1
\end{array}\right)
\end{array}
$$

The last equation can be solved iteratively following the algorithm given in [4]. We consider at the first step of the iteration $\mathcal{W}_{i}(q)^{0}=\left(\begin{array}{l}0 \\ 1\end{array}\right)$.

\section{Validation of stochastic ap- proaches}

We consider that the validation of any stochastic feasibility analysis should always verify the following three conditions:

- Initial condition: if all random variables have an unique value, then the analysis should be able to find the same result as existing deterministic analyses. For instance, let us consider our case where the inter-arrival times are given as random variables. Verifying the initial condition implies that for a system with all random variables having an unique value, i.e. $\mathcal{T}_{i}=$ $\left(\begin{array}{c}T_{i} \\ 1\end{array}\right), \forall i$, the analysis gives us the same answer as the corresponding deterministic analysis.

- Robustness condition: if at least one random variable contains at least two different values, then the analysis should be tested by using rare events theory. Usually the complexity of this type of approach is high (e.g., utilisation of convolutions) and the probability of having very high computations must stay under a certain threshold, i.e., having very high computations must be a rare event.

- Worst-case condition: if at least one random variable contains at least two different values, then the analysis must be able to:

1. deal with systems of periodic and sporadic tasks. The stochastic analysis should be able to answer even when periodic activities exist. 
2. insure the worst-case behaviour of the system. The analysis should be able to detect the instance or the instances having the worst-case response time.

Concerning the analysis we present here, the first condition is not fulfilled, but it allows us to provide the following discussion. When we consider systems with all random variables having an unique value, we obtain the mean of $q$ response times instead the max because of the choice of calculating the average response time in Equation (8). As we said, another way of merging all response times can be used and by applying the initial condition, we can easily detect those that are appropriate.

Concerning the second condition, experimentations are needed in order to conclude on this issue. Once a sufficient number of experimentation exist, one can use theory like Large Deviation [1] to conclude on the robustness of his/her approach.

Concerning the third condition, our approach allows to fulfill only the first part, since our analysis is able to provide answer even when periodic activities exist. The second part of this condition is still an open question in our case except for the case where $\mathcal{T}_{i, j}=\mathcal{T}_{i, k}, \forall j, k$.

\section{Conclusion and future work}

In this paper we extend an existing stochastic analysis from the preemptive uniprocessor case to nonpreemptive CAN case of systems under fixed-priority scheduling. Discussions on validation of this approach were proposed. The approach presented here is a first step to the introduction of dependent random variables for messages scheduled on CAN bus.

Complete validation of our feasibility analysis as mentioned before our approach needs a complete validation by fulfilling all three conditions proposed in Section 5. We need a correct way to merge all response times and to choose the instances that allow us to insure worst-case behaviour.

Case of dependent random variables: introduction of copulas - the introduction of copulas in our analysis is the next logical step. This implies simply replacing the convolutions in Equation ( 8 ) by corresponding operations introduced in [6].

Complexity issues - any approach using convolutions is concerned by its complexity if we want to stay under a certain threshold of pessimism. In our case, a first step to decrease our complexity should be the utilisation of simpler CAN analysis like the one given in [7].

\section{Acknowledgments}

The author would like to thank Mathieu Grenier for helping on CAN related and presentation issues. The author is also thanking anonymous reviewers for their useful comments.

\section{References}

[1] N. Navet, L. Cucu, and R. Schott. Probabilistic estimation of response times through large deviations. WIP session, 28th IEEE Real-Time Systems Symposium (RTSS'07), 2007.

[2] S. Edgar and A. Burns. Statistical analysis of WCET for scheduling. In 22nd of the IEEE RealTime Systems Symposium, 2001.

[3] G. Kaczynski, L. Lo Bello, and T. Nolte. Deriving exact stochastic response times of periodic tasks in hybrid priority-driven soft real-time systems. 12th IEEE International Conference on Emerging Technologies and Factory Automation (ETFA'07), Greece, 2007.

[4] L. Cucu and E. Tovar. A framework for response time analysis of fixed-priority tasks with stochastic inter-arrival times. ACM SIGBED Review, 3(1), 2006.

[5] R.I. Davis, A. Burns, R.J. Bril, and J.J. Lukkien. Controller are network (CAN) schedulability analysis: refuted revisited and revised. RealTime Systems, 35:239-272, 2007.

[6] G. Bernat and M. Newby. Probabilistic WCET analysis, an approach using copulas. Journal of Embedded Computing, 2006.

[7] B. Andersson, N. Pereira, and E. Tovar. A twocompetitive approximate schedulability analysis of CAN. 12th IEEE International Conference on Emerging Technologies and Factory Automation (ETFA'07), Greece, 2007. 